\title{
Gestionar desde la territorialidad: ¿cómo integrar la multifuncionalidad y la participación social en la legitimación del regadío?
}

\author{
Sandra Ricart, Anna Ribas y David Pavón \\ Departamento de Geografía, Universitat de Girona \\ sandra.ricart@udg.edu, anna.ribas@udg.edu,david.pavon@udg.edu
}

\begin{abstract}
Resumen. La estrategia por gestionar el territorio, los recursos naturales y las demandas sociales ha tenido en el desarrollo del regadío un aliado fiel a lo largo de los años. En países como España, Francia e Italia la apuesta por el regadío da fe del vínculo indisociable entre toda actividad económico-productiva del agua y la vertebración del territorio. Regiones como Cataluña, Midi-Pyrénées y Lombardia simbolizan, a su vez, el amplio bagaje cultural del regadío. En esta comunicación se presentan tres casos de estudio (canal Segarra-Garrigues, canal de la Neste y canal de la Muzza) que afrontan la multifuncionalidad del regadío desde políticas, actuaciones y percepciones sociales distintas. A su vez, se presentan las bases de la gestión territorial del regadío como mecanismo de adaptación a la complejidad que supone el trinomio formado por los recursos naturales disponibles, las demandas sociales cambiantes y la vertebración del territorio. La aplicación de dichas bases tiene por finalidad identificar las variables que justifican el (des) acuerdo entre la diversidad de discursos asociados a la promoción del regadío así como determinar el perfil del actor dominante, legitimar el mismo o dilucidar las mejores estrategias y/o acciones para la gestión integrada del agua entre demandas en competencia.
\end{abstract}

Palabras clave: territorio, multifuncionalidad, regadío, legitimación.

\begin{abstract}
The strategy to manage the territory, natural resources, and social demands has been supported by the development of irrigation along the years. In countries like Spain, France or Italy, the commitment on irrigation attests the inseparable link between all economic and productive water use and the structuring of the territory. Regions such as Catalonia, Midi-Pyrenees and Lombardy symbolize, in turn, the broad cultural background of irrigation. In this paper we present three case studies (SegarraGarrigues irrigation canal, Neste irrigation canal, and Muzza irrigation canal) which face multifunctionality differing from irrigation policies, actions, and social perceptions. In turn, the bases of the territorial management of irrigation will be presented as a mechanism to adapt to the complexity to affront the trinomial relationship between availability of natural resources, changing social demands, and the structuring of the territory. The application of those bases is intended to identify variables to justify the (dis) agreement between the diversity of discourses associated with irrigation' promotion, as well as to determine the dominant stakeholder' profile, its degree of legitimacy, or ascertain the best strategies and/or actions for the integrated management of water resources between competing water demands.
\end{abstract}

Keywords: territory, multifunctionality, irrigation, legitimacy.

\section{Introducción}

El agua, como elemento fundamental de desarrollo de la sociedad y su entorno, se sitúa en el centro del debate científico, económico, político y social actual (Antunes et al., 2009). Un debate focalizado en la disponibilidad y la gestión de los recursos hídricos, la compatibilidad entre usos del agua en competencia, el interés creciente por la gestión de los bienes comunes desde la participación social o la intensificación de las demandas de connotación ambiental (Strzepek y Boehlert, 2010). En los últimos cincuenta años, el medio ambiente ha evolucionado desde una posición colateral en las preocupaciones de la sociedad y el contenido de las políticas públicas hasta situarse en el centro de buena parte de los discursos y las percepciones afines a un territorio. Al igual que ha tenido lugar en demás ámbitos de la política pública, tanto el interés por la conservación y la gestión ambiental como la preocupación por el buen estado ecológico de los recursos hídricos han sumado una rele- 
vancia mayor como condicionantes de la dinámica agrícola (Turral et al., 2010). Con ello, el regadío y su gestión han devenido protagonistas de un debate multiescalar y supra temporal donde parte de la sociedad cuestiona sus límites socioeconómicos, sus afecciones ambientales y su repercusión para con el desarrollo rural y la vertebración del territorio, exigiendo con ello una reformulación de la política sectorial precedente (Özerol, 2012). Muchos de los factores que han impulsado el desarrollo del regadío (producción alimentaria, asentamiento de la población, dinamización de la matriz rural) siguen vigentes hoy en día. Sin embargo, el regadío debe adaptarse a un mundo cambiante donde aspectos tales como las demandas hídricas desde actividades recreativas y/o deportivas, los impactos ambientales de su práctica o la legitimidad de la toma de decisiones que superan el interés meramente sectorial generan distintos grados de preocupación social.

\subsection{Las políticas de referencia del regadío}

El agua, el suelo, el medio ambiente, la agricultura, el desarrollo rural o la energía son algunas de las variables sobre las cuales se han definido las políticas sectoriales de ámbito comunitario que condicionan la dinámica y la gestión del regadío (Bossio et al., 2009). Con ello, la Política Agrícola Común, la Directiva Marco del Agua o el Programa de Acción para el Medio Ambiente han focalizado buena parte de los discursos, las acciones y las críticas sobre el binomio formado por la gestión de los bienes comunes y su aprovechamiento para fines eminentemente productivos. Se trata de políticas con tendencia a la contraposición, el conflicto o la rivalidad por mantener la hegemonía en el uso de un recurso específico. Dicha situación ha potenciado la gestión integrada en base a la necesidad de sumar el enfoque transversal a la gestión de la complejidad que conlleva compatibilizar intereses y puntos de vista que difieren en espacio y tiempo.

Desde su origen y mediante adaptaciones de concepto y finalidad, la política europea ha incidido en la vertebración conjunta de aspectos clave para el desarrollo territorial. Con medio siglo de recorrido, los objetivos iniciales de la PAC (proporcionar alimentos a la ciudadanía a precios asequibles y asegurar una renta digna y razonable para los agricultores) siguen vigentes. Sin embargo, el paso del tiempo ha sumado nuevos y complejos retos: la seguridad alimentaria, el cambio climático y la gestión de los recursos naturales o la voluntad de mantener vivo y dinámico el paisaje vertebrado por la economía rural son solo algunos. La primera política comunitaria, aún con dificultades y margen de mejora, se ha configurado como un ejemplo de adaptación a buena parte de los cambios de carácter socioeconómico y ambiental del territorio. En 1992 el comisario europeo MacSharry anunció la reforma considerada, hasta día de hoy, la mayor apuesta de cambio de filosofía de la política agraria, evolucionando desde la política tradicional productivista al considerado enfoque ruralista y multifuncional (Wilson, 2001). La reforma de 1999 (Agenda 2000), la intermedia de 2003 y la revisión médica de 2008 supusieron avances significativos en la línea desarrollada por MacSharry (Upite y Pilvere, 2011; Andrei y Darvasi, 2012). La nueva reforma de la PAC (2014-2020), con el greening como uno de los principales aspectos generadores de debate, se plantea como la garante de un modelo de agricultura acorde con la conservación ambiental con el cual incrementar la producción de alimentos desde la competitividad del sector agrícola y el respeto hacia la biodiversidad del territorio (Horlings y Marsden, 2011).

El crecimiento demográfico, la modernización y expansión del regadío, el desarrollo industrial y las demandas turísticas o la preservación de los sistemas vivos en ríos y humedales han sustentado, así mismo, buena parte de los focos de interés que conlleva la gestión de los recursos hídricos, estructurando el cambio de paradigma entre la vieja y la nueva cultura del agua (Pahl-Wostl, 2007). Dichos paradigmas han definido los distintos enfoques de las 
políticas del agua promovidas a nivel tanto europeo como nacional hasta sustentar las bases de la Directiva Marco del Agua y la propuesta de una perspectiva ecológica y transfronteriza de la gestión del agua, con el objetivo final de obtener un buen estado ecológico de todas las masas de agua para 2015. Una apuesta ambiciosa que recogerá el conocimiento adquirido sobre el funcionamiento de los ecosistemas y que relacionará los mismos con los usos consuntivos del agua (con afectación directa a la agricultura de regadío) así como la inclusión de las problemáticas sociales y culturales en la vertiente económica y ambiental del agua (Anand, 2007).

Se configurará así el impulso por la Gestión Integrada de los Recursos Hídricos (GIRH) en base a la instauración de un enfoque conjunto entre los usos consuntivos y no consuntivos del agua así como de las funciones ambientales del mismo y el fomento de la participación social en temáticas multisectoriales. Desde su acotación inicial en la Conferencia de Naciones Unidas de Mar de Plata (1977), distintos autores han sucumbido al interés de definir su legado: desde aquellos que apuestan por la GIRH como proceso capaz de promover la coordinación de la gestión del agua, el suelo y el medio ambiente de forma equitativa y en beneficio del desarrollo socioeconómico del territorio a quienes conciben la gestión integrada desde la combinación de las tres I (información, instituciones e infrastructura) para con ello asegurar el equilibrio entre las tres $\mathrm{E}$ (equidad, entorno ambiental y economía). Dicho enfoque ha propiciado el impulso de la participación social como elemento inherente de la gestión integrada de los recursos naturales y en respuesta a la aparición y/o intensificación de nuevos actores sociales que defienden la premisa ambiental desde el carácter público y el interés general de la toma de decisiones (Reed, 2008).

Aún con la falta de referencias directas relacionadas con el medio ambiente en los tratados constitutivos del marco europeo, cabe señalar el año 1971 como el año en que las instancias europeas se comprometerán al impulso de la protección ambiental mediante programas de acción específicos. Después de cuatro décadas de implantación, el séptimo PAMA (2013-2020) enfatizará en preceptos como "quien contamina, paga", "cautela y acción preventiva" o "corrección de la contaminación en origen", todos ellos con una significancia plena en el desarrollo de la agricultura y la promoción del regadío.

\subsection{La multifuncionalidad del regadío}

El binomio agua-suelo es esencial para la producción de alimento y también la base que estructura el desarrollo rural, la distribución territorial de la población, la conservación del paisaje y la preservación ambiental (Mata, 2008). Una dualidad entre la generación de bienes privados y funciones públicas (Figura 1). Es decir, la agricultura multifuncional produce bienes tanto privados (alimento, materias primas de origen agrícola o turismo rural) como públicos, los cuales se dividen en sociales (contribución a la viabilidad de las áreas rurales desfavorecidas, la protección de valores culturales y patrimoniales asociados al medio rural o la protección ante el despoblamiento rural) y ambientales (protección de valores paisajísticos, fomento de la biodiversidad y la reducción de los procesos erosivos del suelo).

El concepto de multifuncionalidad vinculado a la práctica agrícola se emplaza en la escena internacional de 1992 en el marco de la Cumbre de la Tierra celebrada en Río de Janeiro, aunque los antecedentes del debate cabe situarlos a mediados de la década precedente. Inicialmente serán dos los enfoques que centrarán su interés en la multifuncionalidad de la agricultura, uno desde la oferta (diversificar las aportaciones productivas de la agricultura) y otro desde la normativa (aportar un marco regulador que incluya las demandas sociales de la agricultura) (Aumand et al., 2006). Con el tiempo, una tercera interpretación de la multi- 
funcionalidad, arraigada a la sociología y la geografía rural, entrará en escena para referirse a un nuevo modelo de agricultura integrado territorialmente en base al uso local de los recursos naturales y el reconocimiento de las demandas sociales (Renting et al., 2003). A nivel europeo, las principales políticas que condicionarán el carácter multifuncional del regadío tenderán, cada vez más, a la adaptación e integración, de forma progresiva, del modelo de agricultura tradicional con las nuevas demandas de gestión integrada de los bienes comunes y la promoción de la legitimidad social en los procesos de toma de decisiones (Gómez-Limón et al., 2002). A resultas y bajo la consideración multifuncional de la agricultura, distintos autores considerarán establecido "un nuevo paradigma unificador con el fin de desarrollar la agricultura postmoderna de acuerdo con las nuevas demandas sociales" (Van Huylenbroeck et al., 2007:5).

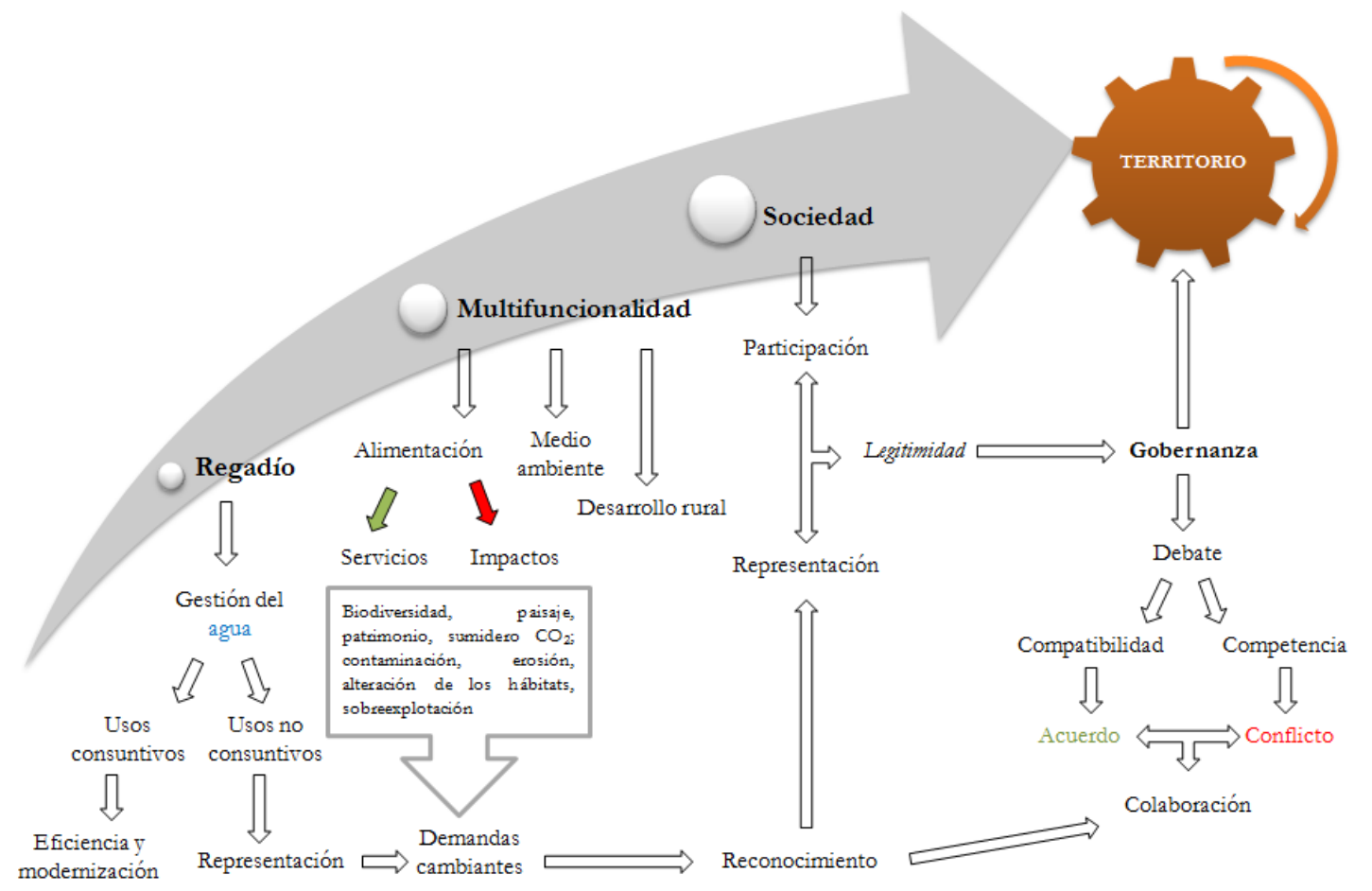

Fig.1. La multifuncionalidad del regadío en la gestión del territorio

Cierto es que la multifuncionalidad, aún con la aparente simplicidad con la que se intuye su descripción, no escapará de la controversia. Así, autores como Dobbs y Pretty (2004) cuestionarán su transposición a nivel político sin demasiados aciertos, mientras otros como Wilson (2001) considerarán la multifuncionalidad de la agricultura (y del regadío) como la transición del carácter productivista de la agricultura a su dimensión sostenibilista. Así, si bien los sistemas de regadío permiten alargar las campañas agrícolas, obtener cultivos durante la estación seca y reducir los daños provocados por las variaciones climáticas, reduciendo con ello la incertidumbre de las demandas productivas y aumentando así mismo la diversidad del mosaico agrícola, también son responsables de actividades y/o actitudes no siempre favorables a la protección de los ecosistemas acuáticos que son motivo de crítica: el (ab)uso de fertilizantes y productos químicos, a la sobreexplotación de acuíferos, la salinización del suelo debido a la mala gestión de los sistemas de regadío o la pérdida de fertilidad del suelo como resultado de la sobreexplotación de suelos frágiles (Hervieu, 2002; Brunstad et al., 2005). Todo ello configura las bases de la multifuncionalidad de la agricultura (y del regadío), tanto a nivel tangible (cantidad y calidad del agua y del suelo utilizados, contribución a la producción de alimento, servicios ambientales generados o mitigación de los efectos acaecidos por el cambio climático) como intangible (valoración y estructuración 
del paisaje, conservación del patrimonio cultural rural o potenciación de las actividades lúdicas).

En este sentido, la apuesta por la práctica agrícola de regadío ha venido (y viene) justificándose, principalmente, mediante cuatro factores: el regadío permite aumentar la productividad del suelo y asegurar con ello la producción alimentaria ante condicionantes climáticos adversos; éste facilita la diversificación de la producción y disminuye la dependencia de la variabilidad del mercado agrícola; así mismo, el regadío da lugar a una mejora de la calidad de los productos y actúa en el margen coste-beneficio de la industria agroalimentaria; y deviene un factor de desarrollo rural y económico de primer orden. Cabe resaltar aquí como el aumento de la población y los cambios dietéticos que pronostican las organizaciones internacionales en materia de alimentación son los principales argumentos a favor del incremento de la producción agrícola de aquí al 2050. Ante perspectiva tal y de forma simplificada, la comunidad científica apuesta por tres acciones: intensificar la productividad agrícola; aumentar la superficie cultivable y/o incrementar la temporalidad del cultivo (cosechas/año) (Khan y Mushtaq, 2009).

Dichos argumentos recibieron, sin embargo, críticas a finales de la década de 1980, cuando algunos autores pronosticaron un aumento de los costes de mantenimiento de los proyectos de regadío así como la intensificación de los condicionantes ambientales y el auge de la oposición social en buena parte de los nuevos proyectos de regadío de cierta envergadura. Así, se viene distinguiendo entre el "valor" del agua (el aprovechamiento para los beneficiarios), el "precio" del agua (el coste para el consumidor) y el "coste de suministro" del agua (coste de inversión, explotación y mantenimiento del sistema). Una distinción asimilada en paralelo a la concepción creciente según la cual los principales retos de la sociedad requieren de un enfoque sostenible en el que el desarrollo se impulse desde la transversalidad entre los aspectos sociales, económicos y ambientales (Sandoval-Solis et al., 2011). Algunos autores apuntarán, por ello, a la imposibilidad de tratar la vertiente económica sin focalizar la atención en la disponibilidad de recursos y la gestión de los límites que la propia naturaleza establece para cada región y ecosistema junto con los aspectos sociales i de gobierno que éstos conllevan (Flora, 2012; Herrick y Pratt, 2012). Todo ello dibujará la balanza entre las externalidades negativas y los servicios ambientales que, a su vez, justificará el carácter multifuncional de la agricultura desde dos enfoques (Hall et al., 2004). Por un lado, desde el carácter productivo del concepto: lejos de acotar-se únicamente a la agricultura, la multifuncionalidad responderá al vínculo entre una actividad y su producto final. Por el otro y en base al marco normativo, la multifuncionalidad responderá a la diversidad de variables asociadas a la consecución de un objetivo específico, con independencia de su naturaleza.

\section{De la gestión tradicional del regadío al enfoque territorial}

La huella dejada por el paso de numerosas civilizaciones, los factores climáticos y orográficos que han condicionado su práctica, los requisitos eminentemente productivos que han estimulado, tradicionalmente, su puesta en marcha, o su adaptación a las demandas ambientales han devenido los principales elementos de estudio en el análisis de la gestión del regadío (Brebbia et al., 2010). Fruto del vínculo difícilmente indisociable entre la disponibilidad hídrica y la actividad agrícola se han configurado unos paisajes resultantes de la mezcla entre la práctica ancestral del regadío tradicional y la apuesta por nuevos proyectos de riego en dominios de secano. Un marco donde conviven sistemas de riego tradicionales que después de un estado de (medio) abandono se ha modernizado con el fin de adaptarse 
a las demandas de carácter ambiental y afianzar con ello la multifuncionalidad del regadío desde su carácter patrimonial y/o lúdico (Dufour et al., 2007).

La gobernabilidad tradicional debe responder a la dificultad creciente que supone reaccionar de forma eficaz a problemáticas complejas por su dinamismo e incertidumbre que cuentan, a su vez, con la atención y/o preocupación de una sociedad cada vez más reflexiva, crítica y exponente de nuevas demandas que no encuentran respuesta en el paradigma tradicional de la gestión del agua (Cairol et al., 2009). Toma fuerza, con ello, la promoción de la gobernanza como mecanismo que favorece la suma de discursos complementarios: el reconocimiento, la aceptación y la integración de la complejidad como elemento intrínseco a la toma de decisiones sobre la gestión de bienes comunes y/o públicos. Un sistema de gobierno mediante el cual la participación y el vínculo entre la pluralidad de partes interesadas permita redistribuir la responsabilidad que conlleva la gestión de recursos naturales tales como el agua y el suelo (Junker et al., 2007). Es por ello que sin compartir dificultades y responsabilidades entre poderes públicos, intereses privados y demandas sociales sea de mayor dificultad avanzar en el cumplimiento de retos transversales en espacio y tiempo. Eso conlleva reconocer al otro, conciliar intereses, equilibrar prioridades y construir compromisos colectivos dentro de un mismo territorio.

\subsection{La gestión tradicional del regadío y su tendencia al compromiso social}

La gestión de la complejidad territorial conlleva, implícitamente, aceptar la evolución de condicionantes tales como la regulación de recursos naturales o la acentuación de conflictos entre usos y funciones del agua. Tradicionalmente, la dinámica del regadío en el ámbito mediterráneo y, más concretamente, sur-europeo, se ha visto influenciada por la convivencia entre a) el mantenimiento de un modelo de gestión heredado del dominio institucional y dirigido, básicamente, a la implicación del sector agrícola como artífice del desarrollo rural, y b) la definición de aquellos vínculos imprescindibles entre las diferentes partes interesadas hasta definir un compromiso capaz de hacer viable el regadío desde la multifuncionalidad y la legitimación social. Con ello, al esquema tradicional de la gestión del regadío, basado en la promoción pública del regadío, su desarrollo infraestructural en colaboración con los intereses privados y el beneplácito de la comunidad rural, se ha incorporado la necesidad de establecer un compromiso social entre las partes para con ello afianzar la convivencia entre la visión marcadamente sectorial del regadío y la concepción que tiende a su socialización de la mano de la gobernanza, es decir, desde su justificación pública. Según Ruf (2004), dicho compromiso social se constituye en base al equilibrio de influencias y/o de poderes dominantes en un territorio ante la posibilidad y/o necesidad de compartir los recursos hídricos disponibles. La propuesta, en 2008, de un Modelo de Compromiso Social del regadío (MCSR) por parte del Institutional and Social Innovations in Irrigation Mediterranean Management (ISIIMM) responde a una nueva forma de afrontar los retos actuales y futuros del regadío mediterráneo. Dicho modelo tiene un doble objetivo: comprender las contradicciones actuales asociadas a la gestión local del agua en las cuencas mediterráneas y proponer innovaciones de carácter institucional capaces de dar respuesta a la realidad social del territorio. Para ello, el MCSR parte de la descripción del regadío desde una doble vertiente. Por un lado, como la elección individual del agricultor que aprovecha las técnicas disponibles para la promoción del regadío como una forma de relacionarse con su entorno. Por el otro, como resultado del desarrollo colectivo que promueve un proyecto de regadío concreto. 


\subsection{Bases para la gestión territorial del regadío}

La mayor parte de los estudios publicados en la actualidad concluyen que la gestión basada en iniciativas de colaboración con una amplia representación de intereses, actitudes y opiniones de los afectado/interesados tienen una probabilidad de éxito mayor que aquellos procesos en los cuales la representatividad es menor (Bidwell y Ryan, 2006). Se busca con ello reafirmar la idea según la cual, ante el reto que supone hacer frente a la complejidad inherente a la gestión de los recursos naturales, los representantes institucionales deben de situarse en paralelo a las demandas de la sociedad. A resultas, las redes sociales están ganando, progresivamente, mayor atención ante la necesidad de gestionar adaptativamente los recursos naturales desde la participación y la cogestión. Autores como Tompkins y Adger (2004) argumentan como las redes sociales entre partes interesadas pueden construir y mejorar la resiliencia de una comunidad e incrementar, con ello, su capacidad de adaptación ante los retos ambientales venideros.

Ante la necesidad de promover una gestión del regadío de carácter territorial capaz de adaptarse, de forma propositiva, a la complejidad que supone el dinamismo del binomio recursos naturales-sociedad, la modelización de la gestión territorial del regadío tiene por objetivo confrontar el conjunto de discursos vinculados al regadío desde una visión transversal en espacio y tiempo. En este sentido, la voluntad de incidir en una gestión de carácter marcadamente territorial del regadío parte de un marco de análisis mayor vinculado a la gestión territorial del agua el cual hace referencia a la organización social de un territorio ante los usos asociados al recurso agua y su distribución entre partes interesadas en competencia desde la planificación participativa y negociada. Se trata, por tanto, de un modelo que a partir de 1) la identificación, aproximación y caracterización de cada una de las partes interesadas en la gestión del canal de regadío desde su discurso, sus postulados y sus preocupaciones; y 2) el análisis de las relaciones de afinidad/confrontación entre discursos y, por tanto, los posicionamientos de los diferentes actores; tiene por finalidad contribuir a la identificación de los aspectos coincidentes/divergentes sobre la gestión del canal de regadío para, con ello, identificar las variables clave en la definición de acuerdos que beneficien al conjunto (Figura 2). En consecuencia, la existencia de un aprendizaje social, la resolución de conflictos latentes o enquistados o la prevención de disputas futuras, la promoción de un plan de acción integrado, la propuesta de soluciones innovadoras o la gestión de las diferencias requerirá de una complicidad entre intereses que en algunos casos comportará hacer compatibles visiones estereotipadas y/o estigmatizadas para poder desencallar un proyecto de regadío en perspectiva de mientras que en otras el foco de atención recaerá en la necesidad de legitimar la toma de decisiones entre una mayor representatividad de intereses (Kallis et al., 2006). 


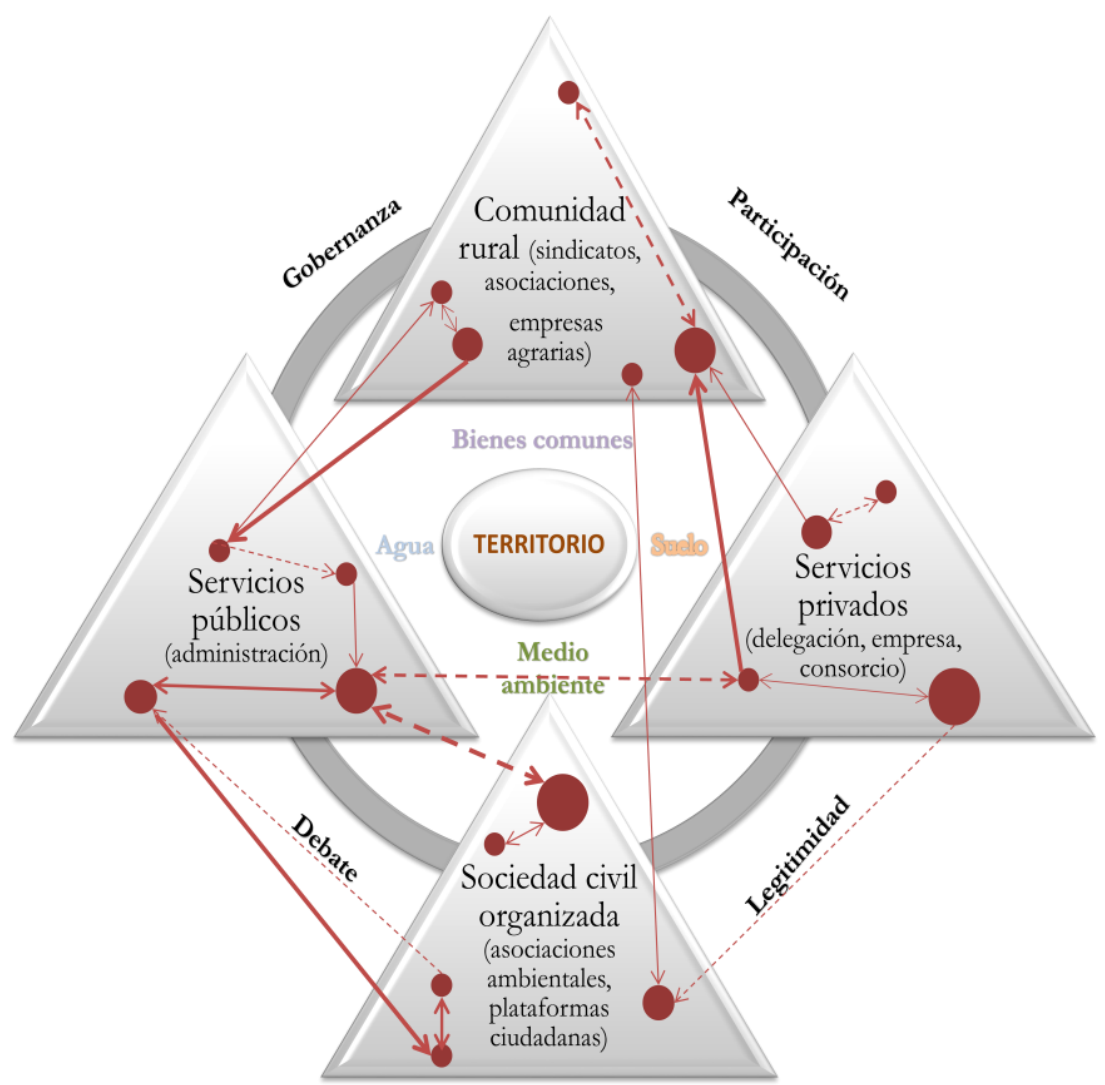

Fig. 2. Red de actores y de vínculos potenciales entre las cuatro categorías de actores implicados en la gestión territorial del regadío

De aquí que el análisis de la gestión territorial del regadío se focalice en lo que se ha identificado como "geografía de actores" o "geografía social", ello es, el análisis de las construcciones territoriales que resultan de los discursos contrapuestos entre la diversidad de actores implicados en la gestión de un recurso natural como el agua y, por extensión, de su incidencia en la dinámica del regadío. Otros autores han relacionado el análisis del territorio desde la "geografía ambiental", es decir, el estudio de las interacciones entre la naturaleza y la sociedad bajo una doble finalidad: comprender la dinámica de los mecanismos sociales ante la gestión de recursos naturales, e interpretar el funcionamiento de los territorios donde estos dominan. Todo ello justifica el uso de dos prácticas de análisis cualitativo, el Stakeholder Analysis Approach y el Governance Model Approach, con el fin de 1) identificar las partes interesadas representativas de la gestión del canal de regadío, 2) confeccionar las bases de su discurso y caracterizar sus demandas mediante un perfil propio y 3) establecer los vínculos de afinidad/confrontación entre los mismos. Todo ello mediante la configuración y realización de entrevistas semi-estructuradas y de cuestionarios on-line una vez completadas las mismas. En cuanto al análisis de su contenido, se ha considerado útil el uso de herramientas de análisis mixto (cualitativo y cuantitativo) que faciliten la exploración de los datos y su posterior tratamiento de forma directa, analítica y comparable. Aquí, el uso del programa Atlas.ti ${ }^{\circledR} 7$ y su habilidad para codificar textos y analizar discursos ha facilitado la tarea de análisis de los discursos de cada uno de los stakeholders, bien desde su identificación (mediante la síntesis de sus citas) bien dese su caracterización y organización (mediante familias y códigos). Todo ello ha favorecido la comparativa posterior de los resultados para con ello radiografiar las variables que generan debate, confrontación y/o acuerdo y perfilar así el actor tipo en cada uno de los ámbitos de estudio. 


\subsection{La gestión territorial del regadío en tres canales de regadío}

Los tres casos de estudio analizados parten de realidades y dinámicas particulares que condicionan las actitudes, las demandas, las críticas, las afinidades y, en definitiva, los discursos contrapuestos que configuran la gestión de los respectivos canales. La representatividad de cada uno de los canales dentro y fuera de su contexto territorial viene definido tanto por los factores coincidentes (el bagaje cultural del regadío; la diversidad de intereses (no) $\mathrm{r}$ presentados; la priorización de unos determinados usos del agua ante la preocupación por su disponibilidad o la participación y/o movilización ciudadana) como los resultados de su contraposición (la recuperación de un proyecto de regadío reivindicado que nace con el paso cambiado, canal Segarra-Garrigues; el debate sobre un canal de regadío que bascula entre el ambientalismo y el monocultivo, canal de la Neste; y la multifuncionalidad de un canal que simula las funciones ambientales de un río, canal de la Muzza). Los tres simbolizan la apuesta por el regadío aunque desde prismas divergentes: de la negación a la colaboración ambiental; de la promoción institucional a la gestión privada; de la competencia a 1 cogestión de usos; de la participación a la legitimidad de la toma de decisiones. Todo ello sintetiza el debate actual y futuro que tiene lugar, con intensidad cambiante, en cada una de las realidades analizadas, situando al regadío en el centro de atención de variables tales como la disponibilidad hídrica y el coste de acceso y uso de los recursos naturales; la priorización de demandas; la estrategia agroalimentaria a nivel regional y/o nacional; las externalidades ambientales que genera la práctica agrícola; o la promoción del desarrollo rural.

2.3.1 El canal Segarra-Garrigues. Proyectado a mediados de la década de 1990 si bien su concepción responde a mediados del s.XIX, los trabajos de construcción se iniciarán en 2002. Con $85 \mathrm{~km}$ de longitud desde el embalse de Rialb y hasta el embalse de L'Albagés, el canal principal agrupa 70.150 hectáreas de superficie, con 73 municipios afectados a lo largo y ancho de seis comarcas: Noguera, la Segarra, l'Urgell, el Pla d'Urgell, les Garrigues y el Segrià (Figura 3). El debate en torno a la disponibilidad de agua y a su prioridad de uso junto con la viabilidad económica de la infraestructura, la constancia del conflicto de intereses entre regantes y ambientalistas (que redujo la superficie destinado al riego inicialmente proyectada hasta menos de la mitad) y la falta de comprensión social del proyecto pondrán en cuestión la legitimidad del mismo (Ricart et al., 2013). El factor que diferencia a dicho canal de los otros dos que se presentarán a continuación es la movilización social que ha generado su puesta en marcha, destacable en dos sentidos (Ricart, 2012). Por un lado, la movilización de carácter ambiental impulsada por las entidades conservacionistas y suscitada alrededor de la declaración de buena parte del ámbito potencial de riego como Zona de Especial Protección para las Aves (ZEPA). Por el otro, el carácter transversal de la movilización social, con el objetivo de impulsar un canal concebido históricamente desde el carácter productivista del agua como un canal que aglutine la diversidad de intereses de la sociedad del siglo XXI y donde la vertebración del territorio sea el factor clave para integrar los valores socioeconómicos, ambientales y culturales del mismo como ejemplo de la multifuncionalidad que fomenta el regadío. 


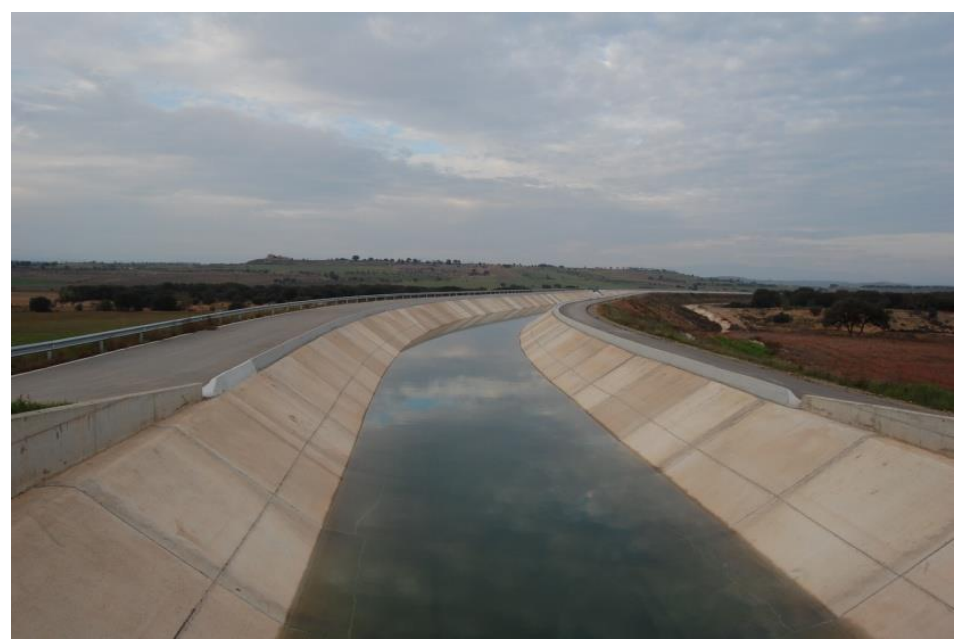

Fig.3. Instantánea del canal Segarra Garrigues a su paso por Verdú (Les Garrigues)

2.3.2 El canal de la Neste. Inaugurado en 1862, este canal de $29 \mathrm{~km}$ conduce las aguas por gravedad desde el río Neste, afluente de la Garona, hasta el valle de Sarrancolin, dentro del Système Neste (Figura 4). Se trata de un complejo hidráulico de diecisiete ríos artificialmente realimentados e interconectados. Además de su función agrícola, juega un rol estratégico al servicio del medio ambiente y de la economía de la región. Desde su concepción, esta obra estatal ha tenido un aprovechamiento multifuncional: drenaje, regadío, navegación, uso hidroeléctrico y uso de boca. Si bien el modelo de gestión del canal viene condicionado por el papel de la CACG como gestor y, en buena medida, como marco de encuentro entre los intereses confrontados, vale la pena resaltar el papel que juega la sociedad civil, donde un solo actor, la asociación ambientalista France Nature Environnement (FNE) capitaliza la respuesta socioambiental frente a la visión más productivista del sector agrario, defendido por el sindicato agrícola dominante, la Fédération nationale des syndicats d'exploitants agricoles (FNSEA). Notoria es, también, la interrelación entre el sector público y la comunidad rural dada la dualidad representativa del regante, ya sea bajo la consideración contractual con la CACG o como miembro de una Association syndicale autorisée d'irrigation (ASA'I) de carácter administrativo y de menor representación territorial. Si bien los usos consuntivos no se han visto modificados de forma importante con el paso del tiempo, cabe destacar el auge que han experimentado los usos no consuntivos del agua asociados al mismo, como la defensa del caudal ecológico o el uso hidroeléctrico (Ricart y Clarimont, 2013). 


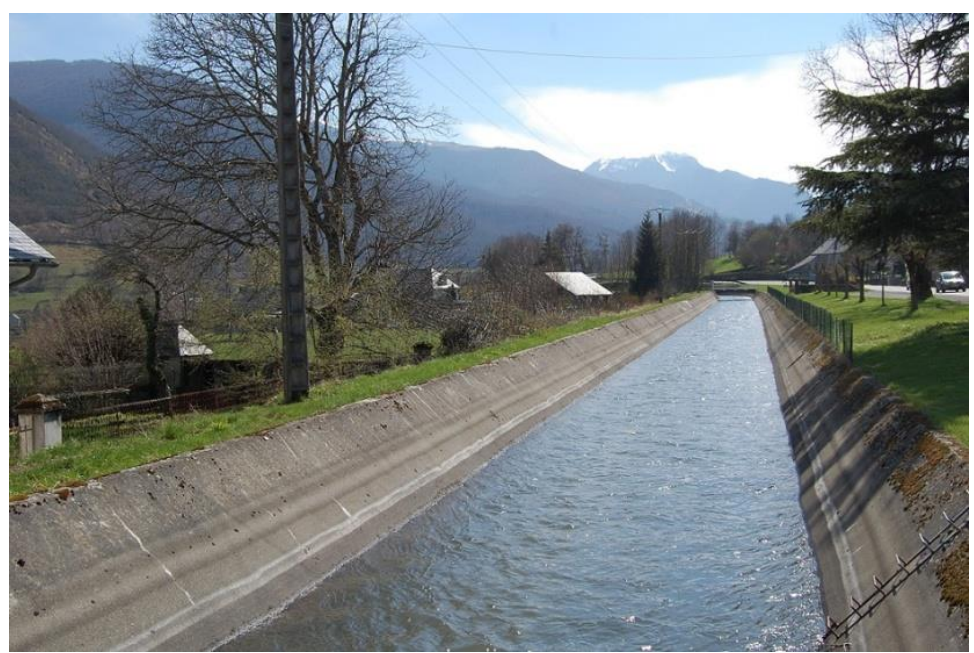

Fig. 4. Instantánea del canal de la Neste a su paso por Hèches (Hautes-Pyrénées)

2.3.3 El canal de la Muzza. Ubicado en el centro de la llanura lombarda, el canal de la Muzza es la obra de referencia de la ingeniería hidráulica del territorio lodigiano (Figura 5). Proyectado en 1220 como derivación del río Adda cerca de Paullo, recorre $61 \mathrm{~km}$ a lo largo de 69 municipios y distribuye el agua a través de cuatrocientos canales secundarios. Promovido con el fin de mejorar las condiciones agronómicas del valle mediante el drenaje de las aguas y la mejora del regadío tradicional, con el paso del tiempo ambas funciones siguen siendo prioritarias pero comparten protagonismo con otras funciones económicas (producción de energía hidroeléctrica y termoeléctrica) y sociales (espacio lúdico y de educación ambiental). Una multifuncionalidad gestionada desde la integración de usos y usuarios en un marco participativo estructurado por el Consorzio dell'Adda y el Consorzio di Bonifica Muzza Bassa Lodigiana. Con su función de mediador entre las partes interesadas en confrontación, el primero gestiona la relación entre los intereses del regadío y los del sector hidroeléctrico desde su potestad de decisión sobre el uso prioritario del agua en cada momento. Por su parte, el segundo gestiona la prioridad de uso del agua (consuntivo o no) entre las demandas presentes en el valle en función de las necesidades del territorio según la época del año.

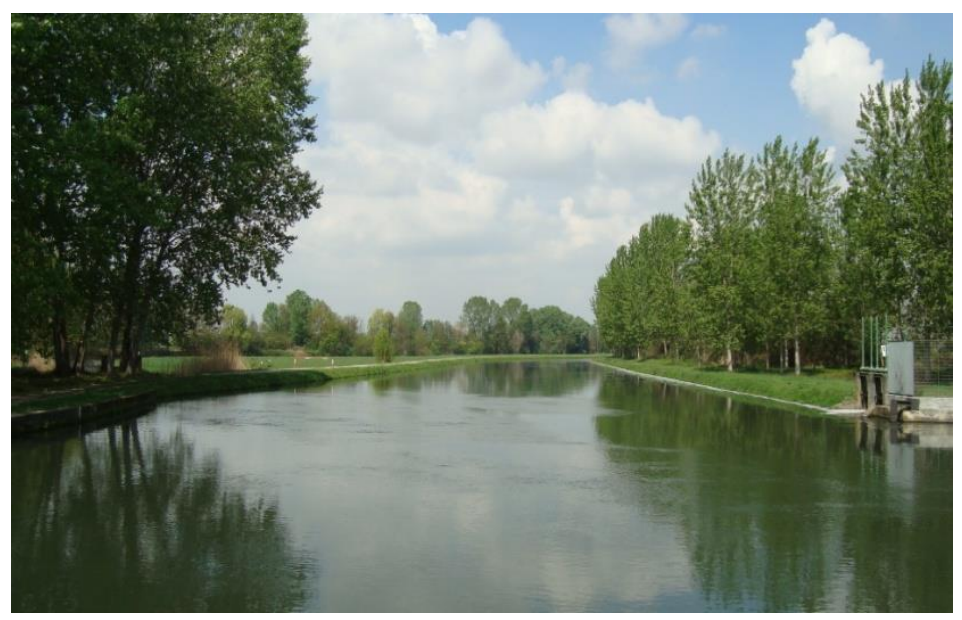

Fig. 5. Instantánea del canal de la Muzza a su paso por Paullo (Milano) 
2.3.4 El perfil del actor dominante en cada uno de los canales de regadío. El estudio de la gestión territorial del canal Segarra-Garrigues ha supuesto la realización de la entrevista y del cuestionario respectivo a un total de 19 actores representativos de los discursos en torno al canal. En total se han contabilizado 411 citas, de las cuales 171 las ha portado la sociedad civil organizada (SEO/BirdLife; Institució de Ponent per a la Conservació i l'Estudi de l'Entorn Natural; Institució per a l'Estudi, Gestió i Recuperació dels Ecosistemes Lleidatans; Lleida Ambiental; Compromís per Lleida; y Plataforma en Defensa de l'Ebre); 126 citas desde la comunidad rural (Unió de Pagesos de Catalunya; Associació Agrària de Joves Agricultors-Associació d'Empresaris Agraris de Lleida; Joves Agricultors i Ramaders de Catalunya; Institut Agrícola Català Sant Isidre; y Federació de Cooperatives Agràries Catalanes); 83 citas por parte de los servicios públicos (Confederación Hidrográfica del Ebro; Agència Catalana de l'Aigua; Departament d'Agricultura, Ramaderia, Pesca, Alimentació i Medi Natural y Subdirecció General d'Infraestructures Rurals i Infraestructures de la Generalitat de Catalunya S.A.U; Oficinas Comarcales del DAAM; y Oficina del Regant); y 31 citas por parte de los servicios privados (Aigües del Segarra Garrigues S.A; Comunitat de Regants del Segarra-Garrigues; y ENDESA).

En el caso del canal de la Neste, se han identificado un total de 11 partes interesadas representativas a quienes se ha entrevistado y enviado el cuestionario respectivo, dando lugar a un total de 203 citas, de las cuales 80 corresponden a aportaciones de los servicios privados (Compagnie d'Amenagement des Coteaux de Gascogne; Syndicat d'Irrigation des Coteaux de Gascogne; Association Syndicale Autorisée La Ribière; y Association Syndicale Autorisee La Basoille); 48 citas provienen de la comunidad rural (Fédération Départemental des Syndicats d'Exploitants Agricoles Hautes-Pyrénées; Confederation Paysanne HautesPyrénées; y Coordination Rurale Hautes-Pyrénées); 43 citas desde la sociedad civil organizada (France Nature Environnement); y 32 citas corresponden a las consideraciones de los servicios públicos (Direction Départementale des Territoires Hautes-Pyrénées; Agence de l'Eau Adour Garonne; y Chambre Départementale d'Agriculture Hautes-Pyrénées).

Por último, el análisis de canal de la Muzza se ha basado en la consideración de 15 partes interesadas representativas a quienes se ha entrevistado y enviado el cuestionario respectivo, dando lugar a un total de 209 citas, de las cuales 67 fueron aportados por los servicios públicos (Regione Lombardia: Ambiente, Energia e Reti; Territorio e Urbanistica; Agricoltura; Sistemi Verdi e Paese; y Autorità di Bacino del Fiume Po); 48 citas por parte de la comunidad rural (Confederazione Generale dell'Agricoltura Italiana Lombardia; Confederazione Nazionale Coldiretti Lombardia; y Confederazione Italiana Agricoltura Lombardia); 48 citas más desde los integrantes de la sociedad civil organizada (WWF Lombardia; Legambiente Lombardia; y Forum Italiano dei Movimenti per l'Acqua); y por último, 46 citas fueron acotadas por los representantes de los servicios privados (Consorzio dell'Adda; Consorzio Bonifica Muzza Bassa Lodigiana; Unione Regionale Bonifiche Irrigazioni e Miglioramenti fondiari Lombardia; y ENEL Lombardia).

El análisis del conjunto de citas acotadas en las entrevistas así como el contenido de las respuestas al cuestionario ha configurado el perfil del actor tipo en cada una de las cuatro categorías de actores representativos de los diferentes canales de regadío (Tablas 1-4). 
Tabla 1. Perfil dominante en los servicios públicos de los tres canales de regadío

\begin{tabular}{|c|c|c|c|}
\hline SERVICIOS PÚBLICOS & $\begin{array}{l}\text { Canal Segarra- } \\
\text { Garrigues }\end{array}$ & Canal de la Neste & $\begin{array}{l}\text { Canal de la } \\
\text { Muzza }\end{array}$ \\
\hline Inclusión en el canal & Complementaria & $\begin{array}{c}\text { Necesaria } \\
\text { Complementaria }\end{array}$ & $\begin{array}{c}\text { Necesaria } \\
\text { Imprescindible }\end{array}$ \\
\hline $\begin{array}{l}\text { ¿Se siente representado en el ca- } \\
\text { nal? }\end{array}$ & Sí & Sí & Sí \\
\hline $\begin{array}{l}\text { ¿En qué consiste dicha represen- } \\
\text { tación? }\end{array}$ & $\begin{array}{c}\text { Formar parte de las } \\
\text { decisiones }\end{array}$ & $\begin{array}{c}\text { Formar parte de las } \\
\text { decisiones }\end{array}$ & $\begin{array}{c}\text { Formar parte de } \\
\text { las decisiones } \\
\text { Influenciar } \\
\text { Presionar } \\
\end{array}$ \\
\hline Nivel de representación & Elevado & Medio - Suficiente & Elevado - Medio \\
\hline $\begin{array}{l}\text { ¿A qué responde su representa- } \\
\text { ción en el canal? }\end{array}$ & $\begin{array}{l}\text { Reconocimiento } \\
\text { institucional }\end{array}$ & $\begin{array}{l}\text { Parte interesada } \\
\text { Reconocimiento } \\
\text { experto }\end{array}$ & $\begin{array}{l}\text { Reconocimiento } \\
\text { institucional }\end{array}$ \\
\hline $\begin{array}{l}\text { Implicación reconocida por parte } \\
\text { del resto de actores }\end{array}$ & $\begin{array}{l}\text { Indispensable } \\
\text { Necesaria }\end{array}$ & $\begin{array}{c}\text { Necesaria } \\
\text { Indispensable }\end{array}$ & $\begin{array}{c}\text { Necesaria } \\
\text { Indispensable }\end{array}$ \\
\hline $\begin{array}{l}\text { Valoración recibida por demás } \\
\text { actores (sobre 5) }\end{array}$ & 2,9 & 2,7 & 3,2 \\
\hline Búsqueda de acuerdos & Sí & No & Sí \\
\hline $\begin{array}{l}¿ D \text { ¿ qué se beneficia el acuerdo } \\
\text { con el resto de actores? }\end{array}$ & $\begin{array}{c}\text { Compatibilidad de } \\
\text { discursos } \\
\text { Facilidad de negociación }\end{array}$ & Estrategia política & $\begin{array}{l}\text { Facilidad de } \\
\text { negociación }\end{array}$ \\
\hline Marco temporal del acuerdo & Permanente & Según temática & $\begin{array}{c}\text { Según temática o } \\
\text { puntual }\end{array}$ \\
\hline Tipo de relaciones dominantes & Bidireccionales & $\begin{array}{c}\text { Solicitud - Recepción } \\
\text { de acuerdo }\end{array}$ & $\begin{array}{l}\text { Recepción de } \\
\text { acuerdo } \\
\text { (unidireccional) }\end{array}$ \\
\hline Factores favorables al acuerdo & $\begin{array}{l}\text { Predisposición a la } \\
\text { colaboración } \\
\text { Reconocimiento como } \\
\text { interlocutor válido } \\
\text { Capacidad de escuchar } \\
\text { Facilidad de negociación } \\
\text { Sinergias a medio plazo }\end{array}$ & $\begin{array}{c}\text { Reconocimiento } \\
\text { como interlocutor } \\
\text { válido } \\
\text { Capacidad de } \\
\text { escuchar } \\
\text { Predisposición a la } \\
\text { colaboración } \\
\text { Sinergias a medio } \\
\text { plazo }\end{array}$ & $\begin{array}{l}\text { Reconocimiento } \\
\text { institucional } \\
\text { Predisposición a la } \\
\text { colaboración }\end{array}$ \\
\hline $\begin{array}{l}\text { ¿Tiene influencia en la gestión del } \\
\text { canal? }\end{array}$ & Sí & Sí & Sí \\
\hline
\end{tabular}


Tabla 2. Perfil dominante en los servicios privados de los tres canales de regadio

\begin{tabular}{|c|c|c|c|}
\hline SERVICIOS PRIVADOS & Canal Segarra-Garrigues & $\begin{array}{l}\text { Canal de la } \\
\text { Neste }\end{array}$ & Canal de la Muzza \\
\hline Inclusión en el canal & Imprescindible & Necesaria & Imprescindible \\
\hline ¿Se siente representado en el canal? & Sí & Sí & Sí \\
\hline $\begin{array}{l}\text { ¿En qué consiste dicha representa- } \\
\text { ción? }\end{array}$ & $\begin{array}{c}\text { Formar parte de las } \\
\text { decisiones }\end{array}$ & $\begin{array}{l}\text { Formar parte de } \\
\text { las decisiones }\end{array}$ & $\begin{array}{l}\text { Marcar tendencia / } \\
\text { ser un referente }\end{array}$ \\
\hline Nivel de representación & Elevado & $\begin{array}{l}\text { Elevado } \\
\text { Mínimo }\end{array}$ & $\begin{array}{l}\text { Elevado } \\
\text { Suficiente }\end{array}$ \\
\hline $\begin{array}{l}\text { ¿A qué responde su representación } \\
\text { en el canal? }\end{array}$ & Reconocimiento social & $\begin{array}{c}\begin{array}{c}\text { Parte interesada } \\
\text { Reconocimiento } \\
\text { institucional }\end{array} \\
\end{array}$ & $\begin{array}{l}\text { Reconocimiento } \\
\text { institucional } \\
\text { Parte interesada } \\
\end{array}$ \\
\hline $\begin{array}{l}\text { Implicación reconocida por parte } \\
\text { del resto de actores }\end{array}$ & $\begin{array}{c}\text { Necesaria } \\
\text { Indispensable }\end{array}$ & $\begin{array}{l}\text { Indispensable } \\
\text { Necesaria }\end{array}$ & $\begin{array}{l}\text { Indispensable } \\
\text { Necesaria }\end{array}$ \\
\hline $\begin{array}{l}\text { Valoración recibida por demás ac- } \\
\text { tores (sobre 5) }\end{array}$ & 2,9 & 2,8 & 2,9 \\
\hline Búsqueda de acuerdos & Sí & No & Sí \\
\hline $\begin{array}{l}\text { ¿De qué se beneficia el acuerdo con } \\
\text { el resto de actores? }\end{array}$ & Facilidad de negociación & & $\begin{array}{c}\text { Compatibilidad de } \\
\text { discursos } \\
\text { Facilidad de } \\
\text { negociación } \\
\end{array}$ \\
\hline Marco temporal del acuerdo & Permanente & & Según temática \\
\hline Tipo de relaciones dominantes & $\begin{array}{l}\text { Recepción de acuerdo } \\
\quad \text { (unidireccional) }\end{array}$ & & $\begin{array}{c}\text { Recepción de } \\
\text { acuerdo (unidirec- } \\
\text { cional) }\end{array}$ \\
\hline Factores favorables al acuerdo & $\begin{array}{l}\text { Facilidad de negociación } \\
\text { Predisposición a la } \\
\text { colaboración } \\
\text { Sinergias a medio plazo }\end{array}$ & $\begin{array}{c}\text { Capacidad de } \\
\text { escuchar } \\
\text { Comprensión del } \\
\text { discurso } \\
\text { Sinergias a medio } \\
\text { plazo }\end{array}$ & $\begin{array}{c}\text { Sinergias a medio } \\
\text { plazo } \\
\text { Reconocimiento } \\
\text { como interlocutor } \\
\text { válido } \\
\text { Facilidad de } \\
\text { negociación }\end{array}$ \\
\hline $\begin{array}{l}\text { ¿Tiene influencia en la gestión del } \\
\text { canal? }\end{array}$ & Sí & Sí & Sí \\
\hline
\end{tabular}


Tabla 3. Perfil dominante en la comunidad rural de los tres canales de regadío

\begin{tabular}{|c|c|c|c|}
\hline COMUNIDAD RURAL & $\begin{array}{l}\text { Canal Segarra- } \\
\text { Garrigues }\end{array}$ & $\begin{array}{c}\text { Canal de la Nes- } \\
\text { te }\end{array}$ & Canal de la Muzza \\
\hline Inclusión en el canal & Necesaria & Necesaria & Imprescindible \\
\hline $\begin{array}{l}\text { ¿Se siente representado en el ca- } \\
\text { nal? }\end{array}$ & No & No & Sí \\
\hline $\begin{array}{l}\text { ¿En qué consiste dicha represen- } \\
\text { tación? }\end{array}$ & & & $\begin{array}{l}\text { Influen- } \\
\text { ciar/presionar }\end{array}$ \\
\hline Nivel de representación & & & Elevado - Medio \\
\hline $\begin{array}{l}\text { ¿A qué responde su (no) repre- } \\
\text { sentación en el canal? }\end{array}$ & $\begin{array}{l}\text { Manca de } \\
\text { reconocimiento }\end{array}$ & $\begin{array}{l}\text { Escasa voluntad } \\
\text { política }\end{array}$ & Parte interesada \\
\hline $\begin{array}{l}\text { Implicación reconocida por parte } \\
\text { del resto de actores }\end{array}$ & $\begin{array}{c}\text { Necesaria } \\
\text { Complementaria }\end{array}$ & $\begin{array}{c}\text { Necesaria } \\
\text { Complementaria }\end{array}$ & Complementaria \\
\hline $\begin{array}{l}\text { Valoración recibida por demás } \\
\text { actores (sobre 5) }\end{array}$ & 2,5 & 1,7 & 2,4 \\
\hline Búsqueda de acuerdos & Sí & No & Sí \\
\hline $\begin{array}{l}\text { ¿De qué se beneficia el acuerdo } \\
\text { con el resto de actores? }\end{array}$ & $\begin{array}{c}\text { Compatibilidad de dis- } \\
\text { cursos } \\
\text { Facilidad de } \\
\text { negociación }\end{array}$ & $\begin{array}{l}\text { Compatibilidad de } \\
\text { discursos }\end{array}$ & $\begin{array}{l}\text { Compatibilidad de } \\
\text { discursos } \\
\text { Estrategia política } \\
\text { favorable } \\
\end{array}$ \\
\hline Marco temporal del acuerdo & Permanente & Permanente & $\begin{array}{c}\text { Según temática o } \\
\text { permanente }\end{array}$ \\
\hline Tipo de relaciones dominantes & $\begin{array}{l}\text { Bidireccionales y } \\
\text { unidireccionales }\end{array}$ & $\begin{array}{l}\text { Recepción de } \\
\text { acuerdo } \\
\text { (unidireccional) } \\
\end{array}$ & $\begin{array}{l}\text { Solicitud de acuerdo } \\
\quad \text { (unidireccional) }\end{array}$ \\
\hline Factores favorables al acuerdo & $\begin{array}{c}\text { Predisposición a la } \\
\text { colaboración } \\
\text { Comprensión del } \\
\text { discurso } \\
\text { Reconocimiento como } \\
\text { interlocutor válido }\end{array}$ & $\begin{array}{c}\text { Sinergias a medio } \\
\text { plazo } \\
\text { Predisposición a la } \\
\text { colaboración }\end{array}$ & $\begin{array}{l}\text { Sinergias a medio } \\
\text { plazo } \\
\text { Predisposición a la } \\
\text { colaboración }\end{array}$ \\
\hline $\begin{array}{l}\text { ¿Tiene influencia en la gestión del } \\
\text { canal? }\end{array}$ & No & Sí & Sí \\
\hline
\end{tabular}


Tabla 4. Perfil dominante en la sociedad civil organizada de los tres canales de regadío

\begin{tabular}{|c|c|c|c|}
\hline $\begin{array}{l}\text { SOCIEDAD CIVIL } \\
\text { ORGANIZADA }\end{array}$ & $\begin{array}{l}\text { Canal Segarra- } \\
\text { Garrigues }\end{array}$ & $\begin{array}{l}\text { Canal de la } \\
\text { Neste }\end{array}$ & Canal de la Muzza \\
\hline Inclusión en el canal & $\begin{array}{l}\text { Imprescindible } \\
\text { Necesaria }\end{array}$ & Necesaria & $\begin{array}{l}\text { Imprescindible } \\
\text { Complementaria }\end{array}$ \\
\hline $\begin{array}{l}\text { ¿Se siente representado en el ca- } \\
\text { nal? }\end{array}$ & No & No & No \\
\hline $\begin{array}{l}\text { ¿A qué responde su (no) represen- } \\
\text { tación en el canal? }\end{array}$ & $\begin{array}{l}\text { Escasa voluntad } \\
\text { política }\end{array}$ & $\begin{array}{l}\text { Escasa voluntad } \\
\text { política }\end{array}$ & $\begin{array}{c}\text { Manca de } \\
\text { reconocimiento } \\
\text { Escasa voluntad po- } \\
\text { lítica } \\
\end{array}$ \\
\hline $\begin{array}{l}\text { Implicación reconocida por parte } \\
\text { del resto de actores }\end{array}$ & $\begin{array}{l}\text { Complementaria } \\
\text { Necesaria }\end{array}$ & $\begin{array}{l}\text { Prescindible } \\
\text { Necesaria }\end{array}$ & $\begin{array}{l}\text { Prescindible } \\
\text { Complementaria }\end{array}$ \\
\hline $\begin{array}{l}\text { Valoración recibida por demás } \\
\text { actores (sobre 5) }\end{array}$ & 2,4 & 2 & 2,3 \\
\hline Búsqueda de acuerdos & Sí & Sí - No & No \\
\hline $\begin{array}{l}\text { ¿De qué se beneficia el acuerdo } \\
\text { con el resto de actores? }\end{array}$ & $\begin{array}{c}\text { Compatibilidad de dis- } \\
\text { cursos } \\
\text { Facilidad de } \\
\text { negociación } \\
\text { Estrategia política }\end{array}$ & $\begin{array}{l}\text { Compatibilidad de } \\
\text { discursos } \\
\text { Facilidad de } \\
\text { negociación } \\
\text { Estrategia política }\end{array}$ & \\
\hline Marco temporal del acuerdo & Permanente & $\begin{array}{l}\text { Puntual - Según } \\
\text { temática }\end{array}$ & \\
\hline Tipo de relaciones dominantes & $\begin{array}{l}\text { Solicitud de acuerdo } \\
\quad \text { (unidireccional) }\end{array}$ & $\begin{array}{l}\text { Solicitud - } \\
\text { Recepción de } \\
\text { acuerdo }\end{array}$ & $\begin{array}{l}\text { Recepción de } \\
\text { acuerdo } \\
\text { (unidireccional) }\end{array}$ \\
\hline Factores favorables al acuerdo & $\begin{array}{c}\text { Reconocimiento } \\
\text { como interlocutor válido } \\
\text { Predisposición a la } \\
\text { colaboración } \\
\text { Capacidad de } \\
\text { escuchar } \\
\text { Comprensión del } \\
\text { discurso } \\
\text { Sinergias a medio } \\
\text { plazo }\end{array}$ & $\begin{array}{c}\text { Reconocimiento } \\
\text { como interlocutor } \\
\text { válido } \\
\text { Capacidad de } \\
\text { escuchar } \\
\text { Sinergias a medio } \\
\text { plazo }\end{array}$ & $\begin{array}{l}\text { Comprensión del } \\
\text { discurso } \\
\text { Predisposición a la } \\
\text { colaboración } \\
\text { Sinergias a } \\
\text { medio plazo }\end{array}$ \\
\hline $\begin{array}{l}\text { ¿Tiene influencia en la gestión del } \\
\text { canal? }\end{array}$ & Sí & Sí & No \\
\hline
\end{tabular}

La configuración de cada uno de los perfiles pone de relieve la dualidad del debate sobre la gestión territorial del regadío desde dos vertientes: la discursiva y la de gestión. En el primer caso, los representantes de los tres canales coinciden en presentar un discurso confrontado entre las demandas productivas vinculadas a la disponibilidad de agua que justifica la comunidad rural con los condicionantes ambientales que antepone la sociedad civil organizada en beneficio del conjunto de la sociedad. Así, la diferencia entre los tres casos de análisis repercute en la intensidad de dicha confrontación, de manera que tanto en el canal Segarra-Garrigues como en el canal de la Neste los posicionamientos presentan mayor visceralidad que lo reconocido para con el canal de la Muzza, donde coexisten ejemplos de colaboración. En cuanto a la gestión práctica del canal, tanto las partes implicadas 
en el canal de la Neste como las respectivas de canal de la Muzza coinciden en destacar la multifuncionalidad del canal.

\section{Conclusiones}

La gestión del territorio, los recursos naturales y las demandas cambiantes de la sociedad ha tenido, en el desarrollo del regadío, un fiel aliado a lo largo del tiempo. La mejora de la productividad agrícola y el reto que supone la soberanía alimentaria; la lucha contra la desertificación; la mitigación del cambio climático y la adaptación a los episodios de escasez hídrica; la compatibilidad con los requerimientos ambientales; o la participación de la sociedad ante actividades que superan el interés meramente sectorial explican buena parte de los debates existentes en torno a la gestión del agua para uso agrícola. Ello ha incrementado la complejidad que envuelve la gestión de los bienes comunes, otorgando a la multifuncionalidad del regadío la capacidad de minimizar la competencia entre usos y demandas en pro de la compatibilidad entre las mismas. Los tres casos de estudio radiografiados coinciden en la necesidad de adaptarse a la incertidumbre que conlleva la variabilidad en la disponibilidad del recurso agua así como a la diversidad de intereses y prioridades presentes en un territorio de por sí complejo. Cada uno aporta, sin embargo, cierta particularidad, ya sea por su propia caracterización (más productivista, ambientalista o integradora), por su enfoque ante la gestión del mismo (sectorial, participativo, en busca de la legitimidad social) y por la naturaleza prioritaria de sus retos futuros (socioeconómicos, ecológicos, políticos). Las bases para la gestión territorial del regadío deben ser de utilidad a la hora de identificar los conflictos latentes y/o potenciales para con ello determinar aquellas acciones capaces de favorecer la multifuncionalidad del regadío desde la participación sectorial y la legitimidad social.

\section{Referencias}

Anand, P. (2007): Right to water and access to water: an assessment, Journal of International Development, 19: 511-526.

Andrei, J-V. y Darvasi, D. (2012): Perspectives and challenges in financing the New Common Agricultural Policy, a new paradigm, Journal of Food, Agriculture \& Environment, 10, 1: 904-907.

Antunes, P. et al. (2009): Participation and evaluation for sustainable river basin governance, Ecological Economics, 68: 931-939.

Aumand, A. et al. (2006): Definitions, references and interpretations of the concept of multifunctionality in France, European Series on Multifunctionality, 10: 5-39.

Bidwell, R. D. y Ryan, C. M. (2006): Collaborative partnership design: The implications of organizational affiliation for watershed partnerships, Society and Natural Resources, 19, 9: 827843.

Bossio, D. et al. (2009): Managing water by managing land: Addressing land degradation to improve water productivity and rural livelihoods, Agricultural Water Management, 97: 536542.

Brebbia, C. A. et al. (2010): Sustainable Irrigation Management, Technologies and Policies III. Southampton: WIT Press. 272 p. 
Brunstad, R. J. et al. (2005): Multifunctionality of agriculture: an inquiry into the complementarity between landscape preservation and food security, European Review of Agricultural Economics, 32, 4: 469-488.

Cairol, D. et al. (2009): Multifunctionality of agriculture and rural areas as reflected in policies: The importance and relevance of the territorial view, Journal of Environmental Policy \& Planning, 11, 4: 269-289.

Dobbs, T.L. y Prretty, J.N. (2004): Agri-Environmental stewardship schemes and "multifunctionality", Review of Agricultural Economics, 26, 2: 220-237.

Dufour, A. et al. (2007): Multifunctionality in Agriculture and its Agents: Regional Comparisons, Sociologia Ruralis, 47, 4: 316-342.

Flora, C. B. (2012): Sustainability unpacked: food, energy and water for resilient environments and societies, Contemporary Sociology, A journal of reviews, 41, 5: 679-681.

Gómez-Limón, J.A. et al. (2002): Conflicting implementation of agricultural and water policies in irrigated areas in the EU, Journal of Agricultural Economics, 53, 2: 259-281.

Hall, C. et al. (2004): What does the public want from agricultura and the countryside? A review of evidence and methods, Journal of Rural Studies, 20: 211-225.

Hervieu, B. (2002): La multifonctionnalité de l'agriculture: genèse et fondements d'une nouvelle approche conceptuelle de l'activité agricole, Cabiers Agricultures, 11, 6: 415-419.

Herrick, C. y Pratt, J. (2012): Sustainability in the water sector: enabling lasting change through leadership and cultural transformation, Nature + Culture, 7, 3: 285-313.

Horlings, L.G. y Marsden, T.K. (2011): Towards the real green revolution? Exploring the conceptual dimensions of a new ecological modernisation of agriculture that could 'feed the world', Global Environmental Change, 21: 441-452.

Junker, B. et al. (2007): Objectives of public participation: which actors should be involved in the decision making of river restorations, Water Resources Research, 43, 10: W10438.

Kallis, G. et al. (2006): Participatory methods for water resources planning, Environment and Planning C: Government and Policy, 24: 215-234.

Khan, S. y Mushtaq, S. (2009): Regional partnerships to assist public-private investments in irrigation system, Agricultural Water Management, 96: 839-846.

Mata, R. (2008): El paisaje, patrimonio y recurso para el desarrollo territorial sostenible. Conocimiento y acción pública, ARBOR, Ciencia, Pensamiento y Cultura, 729: 155-172.

Özerol, G. et al. (2012): Irrigated agriculture and environmental sustainability: an alignment perspective, Environmental Science \& Policy, 23: 57-67.

Pahl-Wostl, C. (2007). The implications of complexity for integrated resources management, Environmental Modelling and Software, 22: 561-569.

Reed, M. S. (2008): Stakeholder participation for environmental management: a literature review, Biological Conservation, 141: 2417-2431.

Renting, H. et al. (2003): Understanding alternative food networks: exploring the role of short food supply chains in rural development, Environment and Planning A, 35, 3: 393-411.

Ricart S. (2012): Vers une gestion territoriale de l'irrigation ? Le canal Segarra-Garrigues (Lleida, Catalogne), en C. Aspe, Ed., De l'eau agricole à l'eau environnementale. Résistance et adaptation aux nowveaux enjeux de partage de l'eau en Méditerranée, Éditions Quae, pp. 337-350. 
Ricart, S. y Clarimont, S. (2013): De la gouvernance appliquée à la gestion de l'irrigation: le cas du canal de la Neste (Hautes-Pyrénées), Sud-Ouest Européen, 35: 69-84.

Ricart S. et al. (2013): La participación en la gestión del regadío como mecanismo para afrontar el conflicto territorial: algunos ejemplos de ámbito sur-europeo, Méditerranée, 120: 73-86.

Ruf, T. (2004): Le système irrigué comme territoire, Territoires en mutation, 12: 51-62.

Sandoval-Solis, S. et al. (2011): Sustainability Index for water resources planning and management, Journal of Water Resources Planning and Management, 137, 5: 381-390.

Strzepek, K. y Boehlert, B. (2010): Competition for water for the food system, Philosophical Transactions of the Royal Society Biological Sciences, 365: 2927-2940.

Tompkins, E. L. y Adger, W. N. (2004): Does adaptive management of natural resources enhance resilience to climatic change?, Ecology and Society, 9, 2: 10.

Turral, H. et al. (2010): Investing in irrigation: Reviewing the past and looking the future, Agricultural Water Management, 97: 551-560.

Upite, I. y Pilvere, I. (2011): The EU Common Agricultural Policy for agricultural and rural development, Management Theory and Studies for Rural Business and Infrastructure Development, 27: 183-190.

Van Huylenbroeck, G. et al. (2007): Multifunctionality of agriculture: A review of definitions, evidence and instruments, Living Reviews in Landscape Research, 1: 3.

Wilson, G. (2001): From productivism to postproductivism ... and back again? Exploring the (un)changed natural and mental landscapes of European agriculture, Transactions of the Institute of British Geographers, 26: 77-102. 